\title{
Myofilament dysfunction in cardiac disease from mice to men
}

\author{
Nazha Hamdani - Monique de Waard · Andrew E. Messer · Nicky M. Boontje · \\ Viola Kooij - Sabine van Dijk · Amanda Versteilen - Regis Lamberts · \\ Daphne Merkus · Cris dos Remedios · Dirk J. Duncker · Attila Borbely · \\ Zoltan Papp - Walter Paulus · Ger J. M. Stienen - Steven B. Marston · \\ Jolanda van der Velden
}

Received: 23 October 2008/ Accepted: 7 December 2008/Published online: 13 January 2009

(C) The Author(s) 2009. This article is published with open access at Springerlink.com

\begin{abstract}
In healthy human myocardium a tight balance exists between receptor-mediated kinases and phosphatases coordinating phosphorylation of regulatory proteins involved in cardiomyocyte contractility. During heart failure, when neurohumoral stimulation increases to compensate for reduced cardiac pump function, this balance is perturbed. The imbalance between kinases and phosphatases upon chronic neurohumoral stimulation is detrimental and initiates cardiac remodelling, and phosphorylation changes of regulatory proteins, which impair cardiomyocyte function. The main
\end{abstract}

N. Hamdani · N. M. Boontje · V. Kooij · S. van Dijk ·

A. Versteilen · A. Borbely · W. Paulus .

G. J. M. Stienen · J. van der Velden $(\varangle)$

Laboratory for Physiology, Institute for Cardiovascular

Research, VU University Medical Center, van der

Boechorststraat 7, 1081 BT Amsterdam, The Netherlands

e-mail: J.vandervelden@vumc.nl

M. de Waard · D. Merkus · D. J. Duncker

Experimental Cardiology, Thoraxcenter, Cardiovascular Research School COEUR, Erasmus MC, University Medical

Center Rotterdam, Rotterdam, The Netherlands

A. E. Messer · S. B. Marston

Cardiac Medicine, National Heart and Lung Institute,

Imperial College London, London, UK

\section{R. Lamberts}

Laboratory for Physiology, Department of Anesthesiology, Institute for Cardiovascular Research, VU University Medical Center, van der Boechorststraat 7, 1081 BT Amsterdam,

The Netherlands

C. dos Remedios

Muscle Research Unit, Institute for Biomedical Research,

The University of Sydney, Sydney, Australia

\section{A. Borbely $\cdot$ Z. Papp}

Institute of Cardiology, UDMHSC, Debrecen, Hungary signalling pathway involved in enhanced cardiomyocyte contractility during increased cardiac load is the $\beta$-adrenergic signalling route, which becomes desensitized upon chronic stimulation. At the myofilament level, activation of protein kinase $\mathrm{A}$ (PKA), the down-stream kinase of the $\beta$-adrenergic receptors $(\beta-\mathrm{AR})$, phosphorylates troponin I, myosin binding protein $C$ and titin, which all exert differential effects on myofilament function. As a consequence of $\beta$-AR downregulation and desensitization, phosphorylation of the PKAtarget proteins within the cardiomyocyte may be decreased and alter myofilament function. Here we discuss involvement of altered PKA-mediated myofilament protein phosphorylation in different animal and human studies, and discuss the roles of troponin $\mathrm{I}$, myosin binding protein $\mathrm{C}$ and titin in regulating myofilament dysfunction in cardiac disease. Data from the different animal and human studies emphasize the importance of careful biopsy procurement, and the need to investigate localization of kinases and phosphatases within the cardiomyocyte, in particular their co-localization with cardiac myofilaments upon receptor stimulation.

Keywords Myofilament function - Phosphorylation . Kinase $\cdot$ Phosphatase, adrenergic signalling ·

Protein kinase A - Heart failure

\section{Introduction}

In healthy humans, the sympathetic nervous system (SNS) as well as the renin-angiotensin-aldosterone system are important mechanisms to maintain adequate perfusion of vital organs via peripheral vasoconstriction, an increase in heart rate and an improvement of myocardial contractility. Although aimed at maintaining cardiac pump function, chronic neurohumoral stimulation in patients with 
cardiovascular disease is detrimental for cardiac function (Packer 1995) illustrated by the negative correlation between noradrenaline plasma levels and prognosis of the patient (Cohn et al. 1984) and by the improvement of symptoms and prolonged survival of patients treated with neurohumoral antagonists (Bohm and Maack 2000).

Activation of the $\beta$-adrenergic receptors by SNS is the main signalling route responsible for increasing cardiomyocyte contractility. Beta-adrenergic receptor ( $\beta$-AR) stimulation induces protein kinase A (PKA)-mediated phosphorylation of L-type $\mathrm{Ca}^{2+}$ channels and ryanodine receptors, increasing cytosolic $\mathrm{Ca}^{2+}$, and of phospholamban, increasing activity of the SR $\mathrm{Ca}^{2+}$-ATPase pump and $\mathrm{SR} \mathrm{Ca}^{2+}$ uptake and loading (Bers 2002). At the myofilament level, PKA-mediated phosphorylation of troponin I (cTnI) and myosin binding protein C (cMyBP-C) decrease myofilament $\mathrm{Ca}^{2+}$-sensitivity (Wolff et al. 1996; Cazorla et al. 2006) and contribute to an acceleration of cardiac relaxation (Solaro et al. 1976; Zhang et al. 1995; Kentish et al. 2001). Stelzer et al. (2007) have proposed a dominant role for $\mathrm{cMyBP}-\mathrm{C}$ phosphorylation in regulating the rate of cross-bridge cycling and force development upon $\beta$-AR stimulation. Moreover, several studies revealed a role for PKA-mediated phosphorylation of titin in regulating cardiomyocyte stiffness (Fukuda et al. 2005; Borbély et al. 2005; Krüger and Linke 2006).

Chronic SNS activation in cardiovascular disease results in down-regulation and uncoupling of $\beta$-adrenergic receptors (Harding et al. 1994; Bristow 2000) and thereby limits cardiac responsiveness during increased cardiac load as occurs during exercise. In human heart failure, down-regulation and uncoupling of the $\beta$-AR pathway leads to decreased PKA-mediated phosphorylation of $\mathrm{Ca}^{2+}$ handling and myofilament proteins. Here we present data from different animal (mouse, rat, pig) and human heart failure models in support for reduced $\beta$-AR-mediated signalling and defects in myofilament function.

\section{Materials and methods}

\section{Cardiac tissue}

All animal experiments were performed in accordance with the guide for the care and use of laboratory ANIMALS (NIH Publication 86-23, revised 1996), and with approval of the Animal Care Committee of the VU Medical Center and of the Erasmus Medical Center. Cardiac samples from the left $(\mathrm{LV})$ or right $(\mathrm{RV})$ ventricular wall were obtained from control and diseased: mouse (myocardial infarction, $\mathrm{MI}$ ), rat (pressure overload), pig (MI) and human (ischemic heart disease, ISHD; idiopathic dilated cardiomyopathy, IDCM) myocardium.

\section{Mice}

In mice (C57B1/6 J of either sex) myocardial infarction $(n=7)$ was produced by permanent ligation of the left anterior descending coronary artery as described before (de Waard et al. 2007). Sham animals $(n=6)$ underwent the operation without infarct induction. Cardiomyocyte measurements and protein analysis were performed in tissue from remodelled LV myocardium.

\section{Rats}

Animals (male, Wistar; body weight $175 \mathrm{~g}$ ) received a single subcutaneous injection of $80 \mathrm{mg} \mathrm{kg}^{-1}$ monocrotaline $(n=10)$ (Lamberts et al. 2007). During its first passage through the pulmonary circulation monocrotaline damages the pulmonary endothelium, thereby inducing pulmonary hypertension, causing right ventricular (RV) hypertrophy resulting eventually in RV heart failure (Leineweber et al. 2000; Korstjens et al. 2002). Sham animals (control, $n=11$ ) received a saline injection. Cardiomyocyte measurements and protein analysis were preformed in isolated skinned cardiomyocytes obtained from RV tissue of quiescent control and failing hearts.

\section{Pigs}

In pigs (2-3 month old Yorkshire-Landrace pigs of either sex) the left circumflex coronary artery was permanently ligated to produce a myocardial infarction $(n=6)$ (van Kats et al. 2000; van der Velden et al. 2004). In sham animals $(n=7)$ the suture was removed. Three weeks after surgery (transmural, $0.5 \mathrm{mg}$ wet weight) needle biopsies were taken from the LV anterior free wall myocardium (in MI pigs: remodelled non-infarcted tissue) and immediately frozen and stored in liquid nitrogen (Zaremba et al. 2007). Cardiomyocyte measurements and protein analysis were performed in the subendocardial part of the biopsies.

\section{Human}

Within the human studies LV tissue samples were obtained during heart transplantation surgery from patients with end-stage ischemic $(n=10)$ and dilated cardiomyopathy $(n=5)$ (NYHA class IV) (Messer et al. 2007; Hamdani et al. 2008). Non-failing heart tissue was obtained from donor hearts $(n=10)$ when no suitable transplant recipient was found. The donors had no history of cardiac disease, a normal cardiac examination, normal ECG and normal ventricular function on echocardiography within $24 \mathrm{~h}$ of heart explantation. The tissue was collected in cardioplegic solution and stored in liquid nitrogen. Samples were 
obtained after informed consent and with approval of the local Ethical Committee (St. Vincents' Hospital Human Research Ethics Committee: file number: H03/118; Title: Molecular Analysis of Human heart Failure).

\section{Cardiomyocyte measurements}

Single cardiomyocytes were obtained via mechanical isolation from left or right ventricular tissue samples, and incubated for $5 \mathrm{~min}$ with Triton X-100 (0.5\%) to remove all membranes. Isometric force was measured at various calcium concentrations $\left(\mathrm{pCa},-\log \left[\mathrm{Ca}^{2+}\right]\right.$, ranged from 4.5 to 9) at $15^{\circ} \mathrm{C}$ and sarcomere length of $2.2 \mu \mathrm{m}$. Figure 1a shows a single human cardiomyocyte glued between a force transducer and a motor. After maximal activation 4-5 measurements were carried out at submaximal $\left[\mathrm{Ca}^{2+}\right]$ followed by a maximal activation. Force values obtained in solutions with submaximal $\left[\mathrm{Ca}^{2+}\right]$ were normalized to the interpolated maximal force values. In a number of cells force measurements were repeated after incubation with the catalytic subunit of protein kinase A (PKA, $40 \mathrm{~min}$; $20^{\circ} \mathrm{C} ; 100 \mathrm{U} / \mathrm{ml}$, Sigma). Force registrations before and after PKA at maximal $\left[\mathrm{Ca}^{2+}\right]$ (pCa 4.5) and submaximal $\left[\mathrm{Ca}^{2+}\right]$ (pCa 5.6) are shown in Fig. 1b. Maximal calcium activated tension (Fmax, i.e., maximal force/cross-sectional area) was calculated by subtracting passive force
(Fpas) from the total force (Ftotal) at saturating $\left[\mathrm{Ca}^{2+}\right]$ (pCa 4.5) (Fig. 1b). Passive tension (Fpas) was determined by shortening the cell in relaxation solution ( $\mathrm{pCa} 9.0)$ by $20 \%$. $\mathrm{Ca}^{2+}$-sensitivity is denoted as $\mathrm{pCa}_{50}$, i.e., $\mathrm{pCa}$ value at which $50 \%$ of Fmax is reached.

In vitro motility assay

Thin filament $\mathrm{Ca}^{2+}$ regulatory function of human troponin was studied with the quantitative in vitro motility assay. Regulation of TRITC-Phalloidin labelled actin (actin- $\Phi$ ) filaments movement over immobilised rabbit fast muscle heavy meromyosin (Fraser and Marston 1995; Messer et al. 2007) was defined as fraction of filaments motile and filament sliding speed. The actomyosin was reconstituted in a flow cell, constructed from a microscope slide and a siliconised coverslip. Actin- $\Phi$ was premixed with tropomyosin and troponin at a $10 \times$ working concentration prior to dilution and infusion into the assay flow cell. Thin filament movement over a bed of immobilised rabbit fast muscle heavy meromyosin $(100 \mu \mathrm{g} / \mathrm{ml})$ was performed and analysed as before (Knott et al. 2002). Affinity chromatography on immobilised monoclonal anti-troponin I antibody was used for purification of the whole troponin complex from human failing (IDCM) and non-failing donor myocardium as described previously (Messer et al. 2007).
Fig. 1 Force measurements in single permeabilized cardiomyocytes. a Single human cardiomyocyte at a sarcomere length of $2.2 \mu \mathrm{m}$. b Isometric force measured at maximal $\left[\mathrm{Ca}^{2+}\right](\mathrm{pCa} 4.5)$ and submaximal $\left[\mathrm{Ca}^{2+}\right]$ (pCa 5.6) before and after protein kinase A in a cardiomyocyte from a patient with heart failure
A

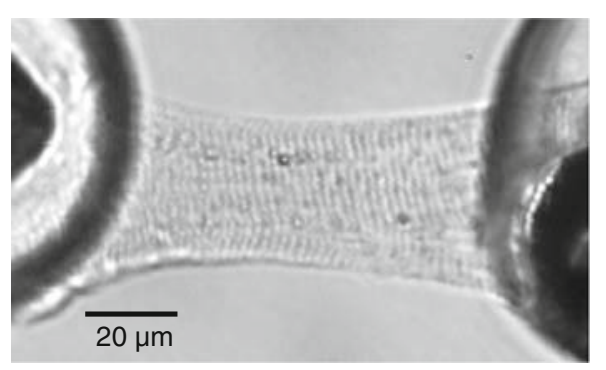

B

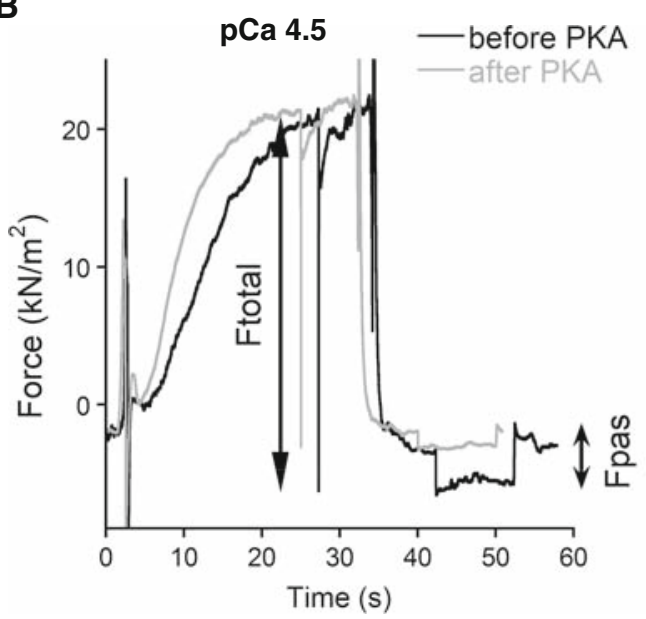

pCa 5.6

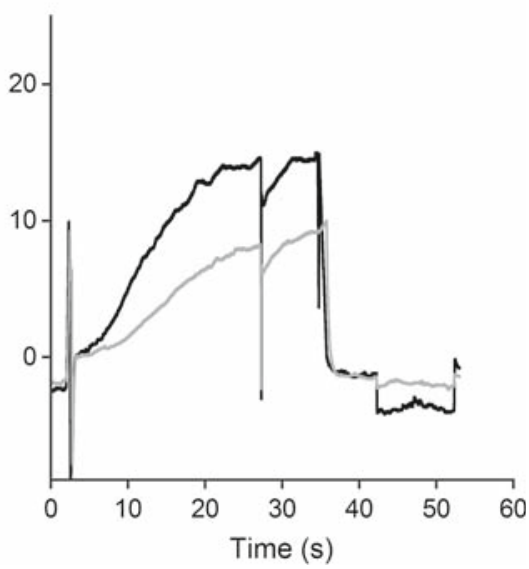


Phosphorylation status of myofilament proteins

\section{ProQ phosphostaining}

Cardiac tissue samples ( 0.5-5.0 mg dry weight) were TCA (tri-chloro acetic acid)-treated as described previously (Zaremba et al. 2007). Phosphorylation status of $\beta$ AR target proteins, myosin binding protein $\mathrm{C}$ (cMyBP-C) and troponin I (cTnI), and other myofilament proteins [desmin, troponin $\mathrm{T}$ (cTnT) and myosin light chain 2 (MLC2)] was determined using Pro-Q Diamond phosphostaining (Molecular Probes). Samples were separated on a gradient gel (Criterion Tris-HCl 4-15\% gel, BioRad) and proteins were stained for $1 \mathrm{~h}$ with Pro-Q diamond phosphoprotein stain. Fixation, washing and de-staining were performed according to the manufacturers guidelines. Staining was visualized using the LAS-3000 Image Reader (FUJI; 460/605 nm Ex/Em; 2 min illumination) and signals were analyzed with AIDA. All protein signals were within the linear range. Subsequently gels were stained overnight with SYPRO Ruby stain (Molecular Probes) and visualized with the LAS-3000 (460/605 nm Ex/Em; 2 s illumination). The phosphorylation signals of myofilament proteins were normalized to SYPRO-stained cMyBP-C to correct for minor differences in protein loading.

\section{Western immunoblotting}

Gel electrophoresis (15\% acrylamide gels) and Western immunoblotting was performed to analyze bisphosphorylation of cTnI at Ser-23/24 (i.e., PKA-sites, rabbit polyclonal $\mathrm{Ab}$, dilution 1:500, Cell signalling). All signals were normalized to Ponceau-stained actin to correct for differences in protein loading.

\section{Statistical analysis}

Data are given as means \pm SEM. Data of control and failing animals/human were compared using unpaired Student $t$-tests. Effects of PKA were tested by paired student $t$-tests. The differences in $\mathrm{pCa}_{50}$ between groups were tested with one-way analysis of variance (ANOVA). Significance was accepted when $P<0.05$. All protein signals were visualized using the LAS-3000 image reader (FUJI) and signals were analyzed with AIDA software (Raytest).

\section{Results}

Enhanced myofilament $\mathrm{Ca}^{2+}$-sensitivity in cardiac disease

Force measurements in single cardiomyocytes revealed an increase in myofilament $\mathrm{Ca}^{2+}$-sensitivity in diseased (failing) myocardium in comparison to control/sham (nonfailing) hearts. Figure $2 \mathrm{a}$ illustrates a leftward shift of the force-pCa relationship in all failing models. The difference in $\mathrm{pCa}_{50}$ between failing and non-failing tissue differed significantly among groups ( 1 -way ANOVA, $P<0.001$ ) and was smallest in the mouse myocardial infarction model $\left(\Delta \mathrm{pCa}_{50}=0.05 \pm 0.01\right)$, and amounted to $0.07 \pm 0.01$ in rat, $0.12 \pm 0.01$ in pig and $0.14 \pm 0.02$ in human (Fig. 2c).

Correction of enhanced myofilament $\mathrm{Ca}^{2+}$-sensitivity by exogenous protein kinase $\mathrm{A}$

Cardiomyocyte force measurements were repeated after incubation with exogenous protein kinase A. Consistent with previous studies, PKA reduced $\mathrm{Ca}^{2+}$-sensitivity of the myofilament in all samples (van der Velden et al. 2003; Édes et al. 2008). This is illustrated by the reduction in force development after PKA at submaximal $\left[\mathrm{Ca}^{2+}\right](\mathrm{pCa}$ 5.4) in Fig. 1b. Incubation with PKA abolished the difference in baseline $\mathrm{Ca}^{2+}$-sensitivity in all groups (Fig. 3a). The reduction in $\mathrm{pCa}_{50}$ was significantly larger in failing compared to non-failing hearts in all models (Fig. 3b). Among the failing groups the shift in $\mathrm{pCa}_{50}\left(\Delta \mathrm{pCa}_{50}\right)$ was smallest in MI mice and largest in end-stage failing human cardiomyocytes.

\section{Altered thin filament regulation}

To study if changes in troponin phosphorylation account for altered contractile function, $\mathrm{Ca}^{2+}$-regulation of reconstituted thin filaments was studied using quantitative in vitro motility assay. $\mathrm{Ca}^{2+}$-regulation of actin motiltity by troponin was studied comparing troponin from failing (IDCM) and non-failing (donor) human myocardium. In Fig. 4a thin filament sliding speed and motile fraction are plotted as a function of $\left[\mathrm{Ca}^{2+}\right]$ (i.e., pCa). Failing heart thin filament $\mathrm{Ca}^{2+}$-sensitivity was always significantly higher than non-failing for both sliding speed $\left(\Delta \mathrm{pCa}_{50}=0.39\right)$ and fraction motile $\left(\Delta \mathrm{pCa}_{50}=0.38\right) \cdot \mathrm{Ca}^{2+}$-sensitivity of thin filament regulation was decreased when non-failing troponin was reconstituted with recombinant PKA-phosphorylated human cTnI (not shown; Messer et al. 2007). When failing and non-failing donor troponin were reconstituted with recombinant human cTnI phosphorylated with PKA, $\mathrm{Ca}^{2+}$-regulation of thin filaments was similar for failing and non-failing troponin (Fig. 4b).

Reduction in maximal force generating capacity in cardiac disease

While force generation at low calcium concentrations was higher in cardiomyocytes from failing compared to nonfailing hearts, the maximal force generating capacity was 
Fig. $2 \mathrm{Ca}^{2+}$-sensitivity of myofilaments in different species. a $\mathrm{Ca}^{2+}$-sensitivity of the myofilaments was significantly higher in failing compared to non-failing controls in all studies. b A reduction in maximal force generating capacity was observed in post-infarct remodelled myocardium. $\mathbf{c} \dagger$ $P<0.05$, animal versus human in Bonferroni post-test analysis
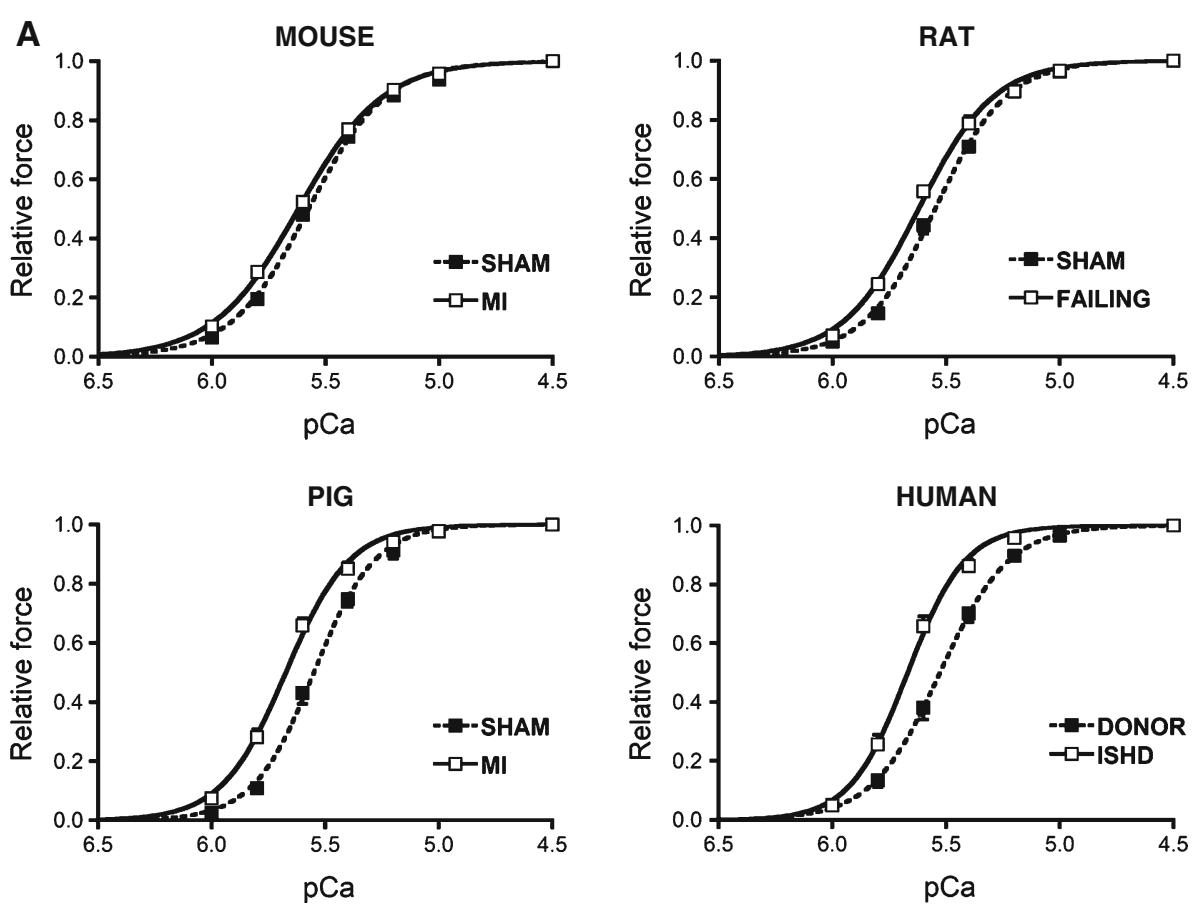

B

PIG

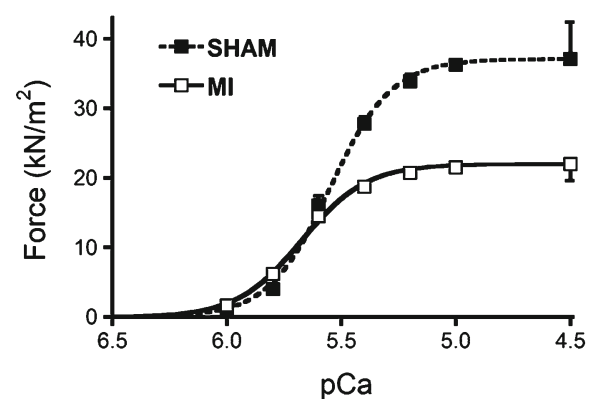

C

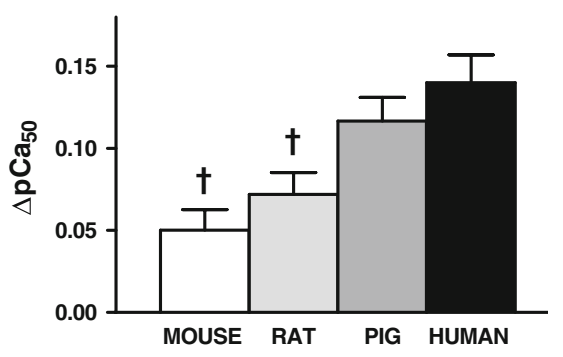

significantly lower in the infarct mouse (sham: $18.5 \pm 1.8 \mathrm{kN} / \mathrm{m}^{2}$ and MI: $14.6 \pm 1.1 \mathrm{kN} / \mathrm{m}^{2}$ ) and pig model. Figure $2 \mathrm{~b}$ illustrates the absolute force values plotted as function of $\mathrm{pCa}$ in the pig model, illustrating the reduction in myofilament force development in cardiomyocytes from post-infarct remodelled myocardium. PKA did not abolish the difference in Fmax (not shown). Fmax did not differ in the failing rat model (control: $24.4 \pm 2.6 \mathrm{kN} / \mathrm{m}^{2}$ and failing: $29.0 \pm 2.1 \mathrm{kN} / \mathrm{m}^{2}$ ) and between human ISHD $\left(33.1 \pm 3.0 \mathrm{kN} / \mathrm{m}^{2}\right)$ and donor $\left(33.8 \pm 2.5 \mathrm{kN} / \mathrm{m}^{2}\right)$ cardiomyocytes.

Correction of enhanced cardiomyocyte stiffness by protein kinase $\mathrm{A}$

Passive force did not significantly differ between cells from failing and non-failing hearts in the mouse, rat and pig model, while cardiomyocyte stiffness was significantly lower in ISHD compared to non-failing donor (Fig. 5a). PKA reduced Fpas in all models. Noteworthy, recent studies in cardiomyocytes isolated from human biopsies (Borbély et al. 2005; van Heerebeek et al. 2006), which were taken during cardiac catheterization revealed enhanced cardiomyocyte stiffness in patients with heart failure. The Fpas was significantly lowered upon incubation with PKA. Figure 5b shows Fpas in cardiomyocytes from heart failure patients with reduced left ventricular ejection fraction (HFREF; NYHA II-III) in comparison to controls. In both HFREF and controls PKA significantly reduced cardiomyocyte stiffness. The reduction in Fpas upon PKA is also illustrated in Fig. 1 b.

Phosphorylation status of myofilament proteins

ProQ Diamond staining of a pig sample incubated with and without PKA showed increased phosphorylation of the $\beta$ AR myofilament target proteins, cMyBP-C and cTnI, while MLC2 phosphorylation was decreased during the incubation (Fig. 6a). ProQ Diamond stained samples from mouse, rat, pig and human samples are shown in Fig. 6 b. 
Fig. 3 Effect of protein kinase A on myofilament $\mathrm{Ca}^{2+}$ sensitivity. a Force-pCa relations after incubation with PKA. After PKA myofilament $\mathrm{Ca}^{2+}$-sensitivity did not differ between failing and non-failing cardiomyocytes. b Change in $\mathrm{pCa}_{50}\left(\Delta \mathrm{pCa}_{50}\right)$ upon PKA in all groups. $* P<0.05$, failing versus non-failing in unpaired student $t$-test
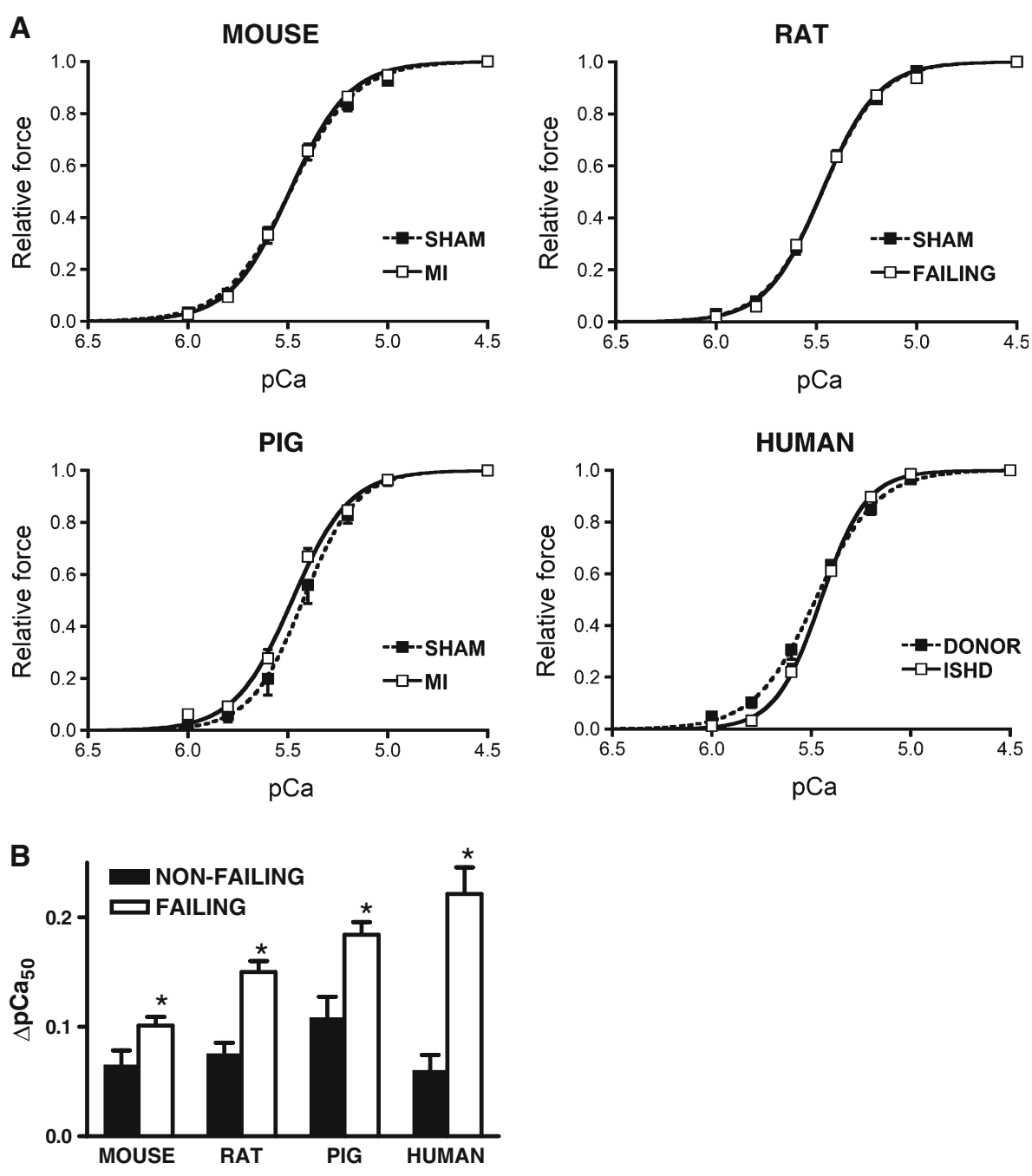

Phosphorylation signals were normalized to the SYPROstained cMyBP-C band in the same samples to correct for small differences in protein loading. Moreover, to correct for differences in staining between gels the PeppermintStick Phosphoprotein marker (PPM, Molecular Probes) was used as described previously (Zaremba et al. 2007; see PPM in Fig. 7). This marker contains phosphorylated ovalbumin. The ProQ Diamond signal for ovalbumin is divided by the SYPRO-stained ovalbumin band, which is subsequently used as correction factor for inter-gel differences.

Analysis of the average values for myofilament protein phosphorylation, obtained after correction for protein loading and inter-gel differences, revealed reduced phosphorylation of cMyBP-C and cTnI in failing human compared to non-failing donor myocardium (Fig. 6c). No significant changes in myofilament protein phosphorylation were observed between failing and non-failing mouse and rat samples. In pig myocardium, MLC2 phosphorylation was significantly lower in MI compared to sham. A similar reduction in MLC2 phosphorylation was observed in failing rat hearts compared to shams, although the difference was not significant $(P=0.08)$. In contrast, MLC2 phosphorylation was significantly higher in end-stage human ISHD than in non-failing donor hearts.

Noteworthy, phosphorylation of cTnI was much higher in mice cardiac samples in comparison to myocardium from other species, in particular pig and human. To determine if PKA is able to increase cTnI phosphorylation in human myocardium to values observed in mice myocardium, a donor and failing human sample were treated with exogenous PKA. The gels shown in Fig. 7 illustrate that the effect of PKA on protein phosphorylation was only minor in the non-failing donor sample $(5 \%$ increase in cMyBP-C, no effect on cTnI), while PKA increased cMyBP-C and cTnI phosphorylation in the failing sample by 27 and $130 \%$, respectively. These data show that PKAphosphorylation in non-failing donor myocardium is almost saturated. The relatively high phosphorylation status of cTnI observed in particular in mouse myocardium 

filaments by human troponin. $\mathrm{Ca}^{2+}$-regulation of thin filament motility by non-failing and failing heart troponin was compared in dual chambered motility cells. a Thin filament failing than non-failing troponin, both for sliding speed and fraction motile. $\mathbf{b}$ The difference was abolished when failing and non-failing troponin were reconstituted with PKAphosphorylated recombinant human cTnI
Fig. $4 \mathrm{Ca}^{2+}$-regulation of thin $\mathrm{Ca}^{2+}$-sensitivity was higher for
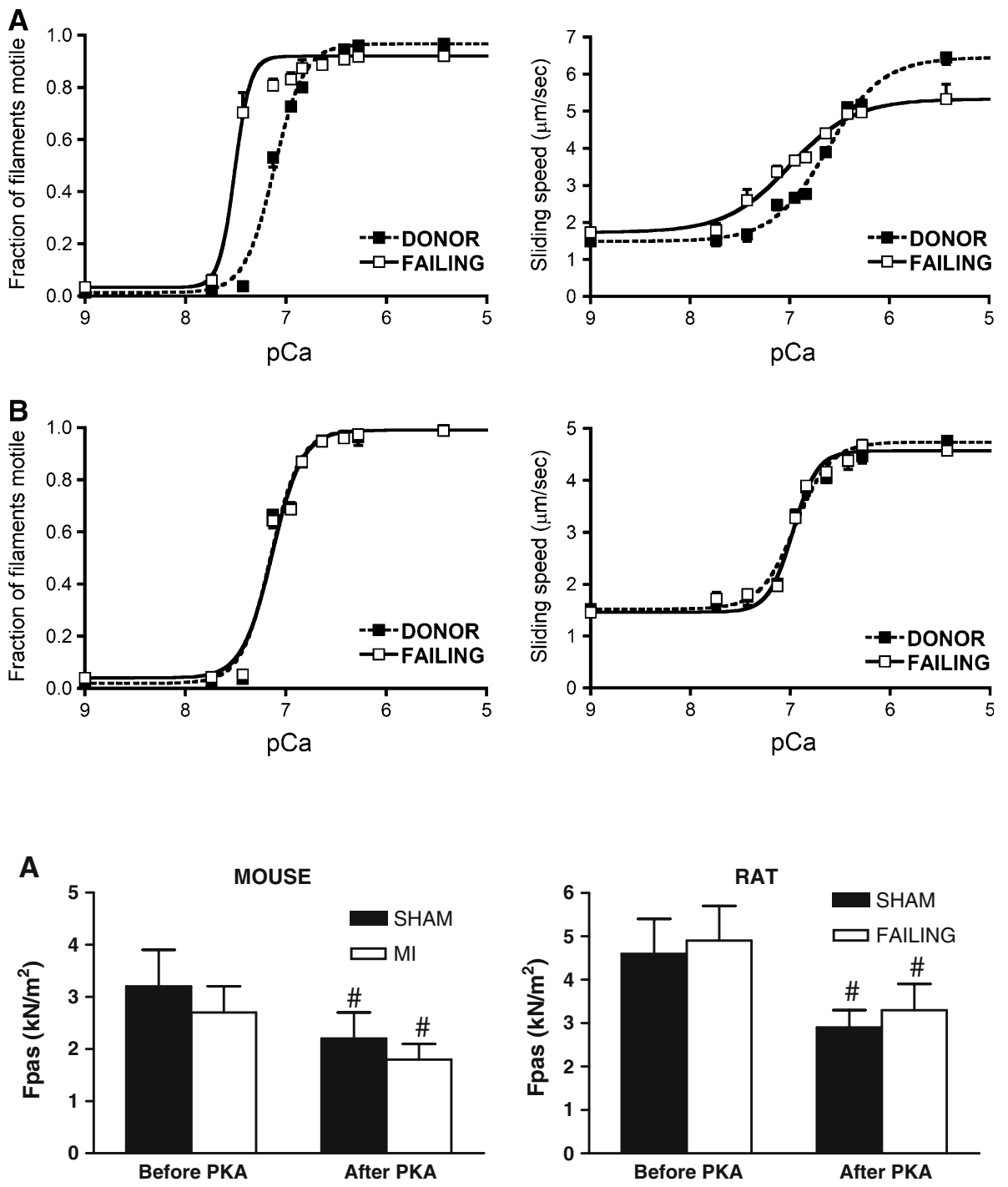

Fig. 5 Cardiomyocyte stiffness. a Protein kinase A (PKA) reduced cardiomyocyte passive force (Fpas). b Force measurements in cardiomyocytes from human cathether biopsies revealed enhanced cardiomyocyte stiffness in heart failure patients with reduced left ventricular ejection fraction (HFREF) compared to controls. $* P<0.05$, failing versus nonfailing in unpaired student $t$-test; ${ }^{\#} P<0.05$, effect PKA in paired student $t$-test
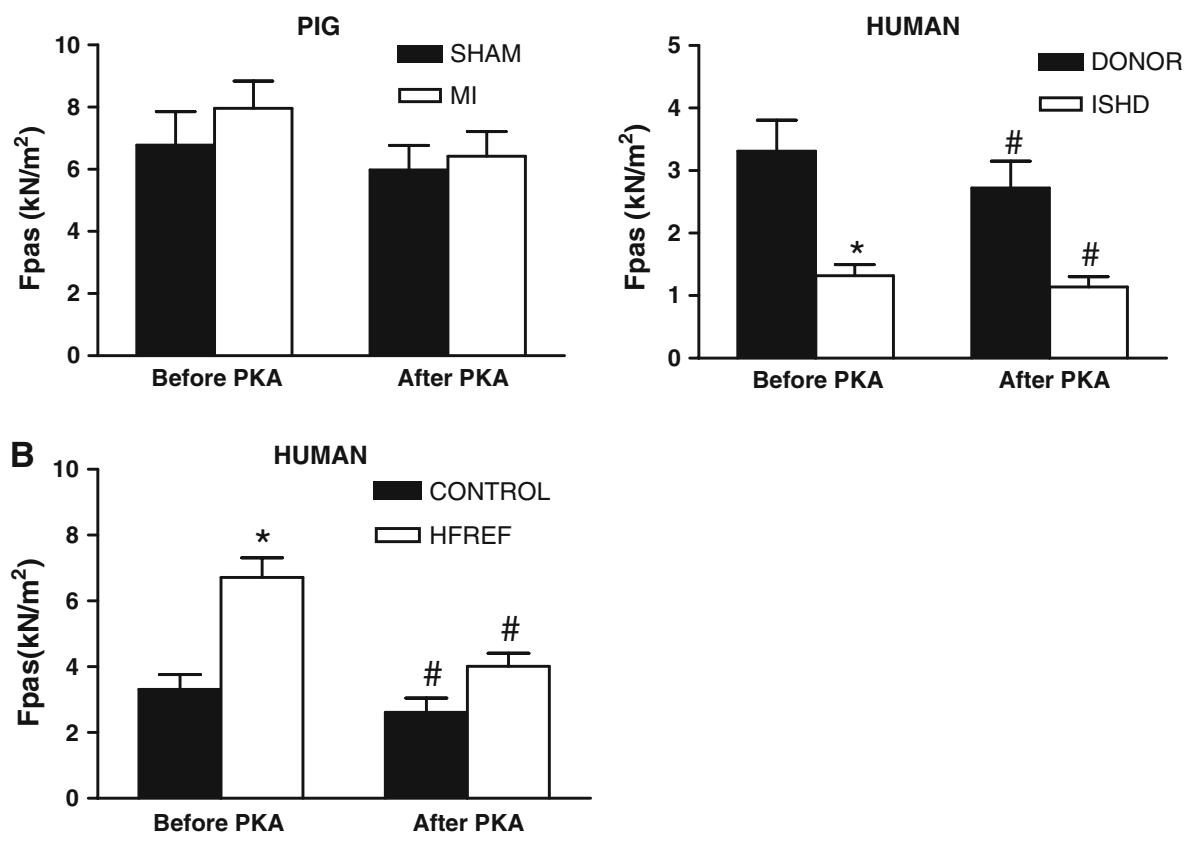


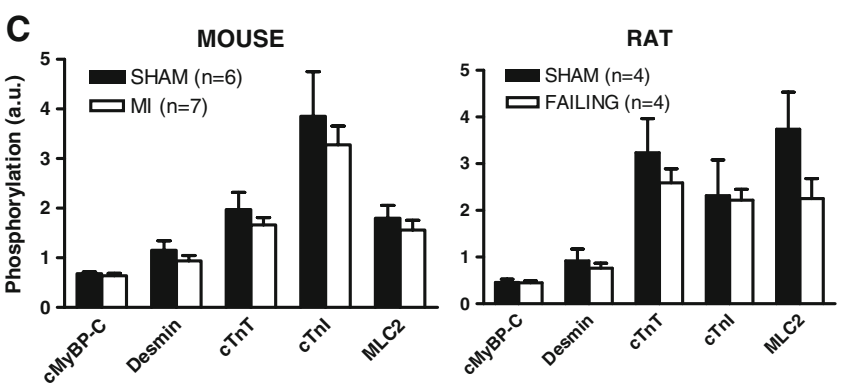

Fig. 6 ProQ-phosphostaining of myofilament proteins. a Pig samples treated with and without PKA, stained with ProQ diamond. b ProQ diamond staining of cardiac samples from mouse, rat, pig and human cardiac samples. Mean values for protein phosphorylation (relative to SYPRO-stained cMyBP-C) are given in c Abbreviations: $M H C$
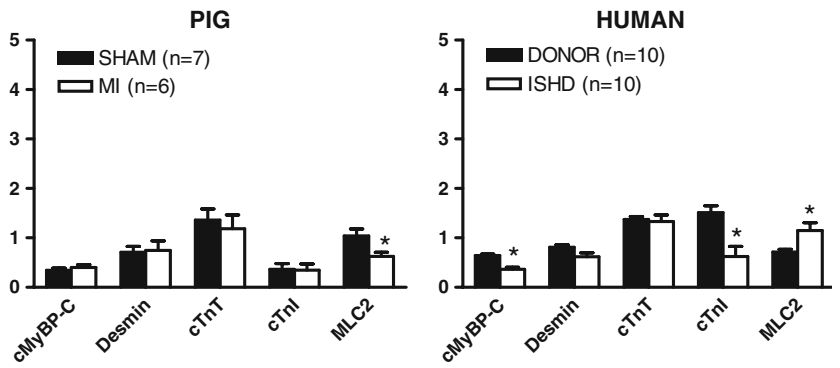

myosin heavy chain; $c M y B P-C$ myosin binding protein $\mathrm{C} ; c \operatorname{Tn} T$ troponin T; $c T n I$ troponin I; $M L C 2$ myosin light chain 2; $F$ failing; $D$ donor; ISHD ischemic heart disease. $n$ number of heart samples. $* P<0.05$, failing versus non-failing in unpaired student $t$-test

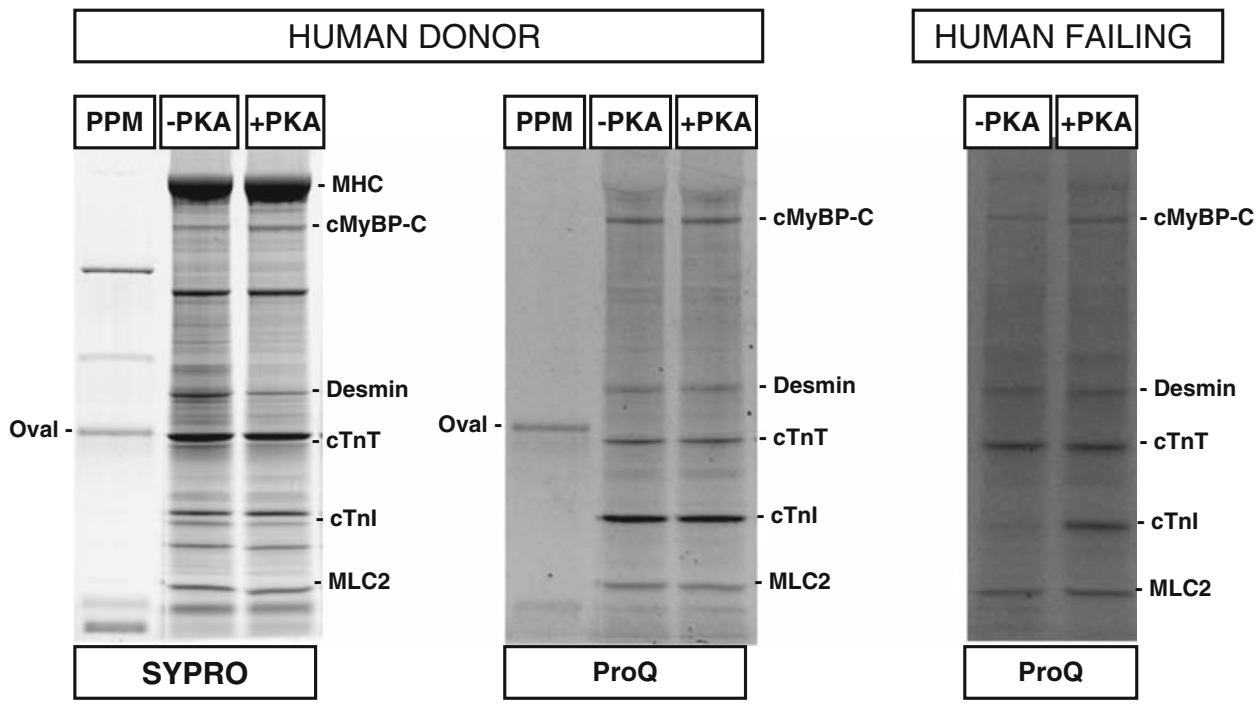

Fig. 7 SYPRO and ProQ-phospho staining of human cardiac samples treated with PKA. PKA incubation of a donor sample slightly increased cMyBP-C, but did not increase cTnI phosphorylation, while an increased ProQ-staining of cMyBP-C and cTnI was observed in failing myocardium treated with PKA. Please note that MLC2 phosphorylation was preserved during PKA treatment as the specific PP1 inhibitor, calyculin A, was present during incubation. Abbreviations: $P P M$ PeppermintStick Phosphoprotein marker; oval ovalbumin; $M H C$ myosin heavy chain; $c M y B P-C$ myosin binding protein $\mathrm{C} ; c T n T$ troponin $\mathrm{T}$; $c T n I$ troponin I; MLC2 myosin light chain 2 
might reflect phosphorylation at other sites, possibly targeted by $\mathrm{PKC}$, and may be related to differences between species and/or tissue handling.

Western immunoblot analysis of cTnI phosphorylation at serines 23/24 revealed significantly lower phosphorylation at the PKA-sites of cTnI in ISHD compared to nonfailing donor myocardium (Fig. 8a, b), while no significant differences were observed in the animal models. It should be noted that the antibody only detects the PKA-bisphosphorylated form of cTnI and not mono-phosphorylated cTnI forms. As our analysis cannot distinguish between mono-phosphorylation of PKA-sites, a difference in the mono-phosphorylated forms of cTnI may still be present between failing and non-failing myocardium.

\section{Discussion}

Activation of the $\beta$-AR pathway results in enhanced cardiac contractile performance in response to increased circulatory demand. However, reduced responsiveness to $\beta$-AR stimulation in heart failure is thought to limit cardiac performance during exercise. Functional data from all our animal and human studies are in support of reduced $\beta$-AR signalling, as baseline myofilament dysfunction (i.e., enhanced $\mathrm{Ca}^{2+}$-sensitivity and cardiomyocyte stiffness) was restored upon incubation with exogenous protein kinase A. However, analysis of the phosphorylation status of $\beta$-AR target proteins revealed reduced PKA-mediated phosphorylation only in end-stage failing human myocardium. Moreover, the reduction in maximal force generating capacity was only observed in post-infarct remodelled myocardium early after MI (mouse and pig model), and could not be restored by PKA. The discrepancies in myofilament function and protein phosphorylation are discussed below.

Enhanced myofilament $\mathrm{Ca}^{2+}$-sensitivity and stiffness

In healthy myocardium, myofilament $\mathrm{Ca}^{2+}$-sensitivity is reduced upon $\beta$-AR stimulation and is thought to exert a positive lusitropic effect on cardiac performance (Kobayashi and Solaro 2005). The reduction in myofilament $\mathrm{Ca}^{2+}$-sensitivity has been ascribed to PKA-mediated phosphorylation of cTnI and cMyBP-C (Wolff et al. 1996; Cazorla et al. 2006). As a consequence of reduced $\beta$-AR
Fig. 8 PKA-mediated cTnI phosphorylation. a Western immunoblot analysis revealed reduced PKA-mediated phosphorylation at Ser 23/24 in cTnI in ISHD compared to nonfailing human myocardium. b Phosphorylation signals of cTnI were normalized to Ponceau-stained actin to correct for differences in protein loading. $n$ number of heart samples. $* P<0.05$, ISHD versus donor in unpaired student $t$-test
A
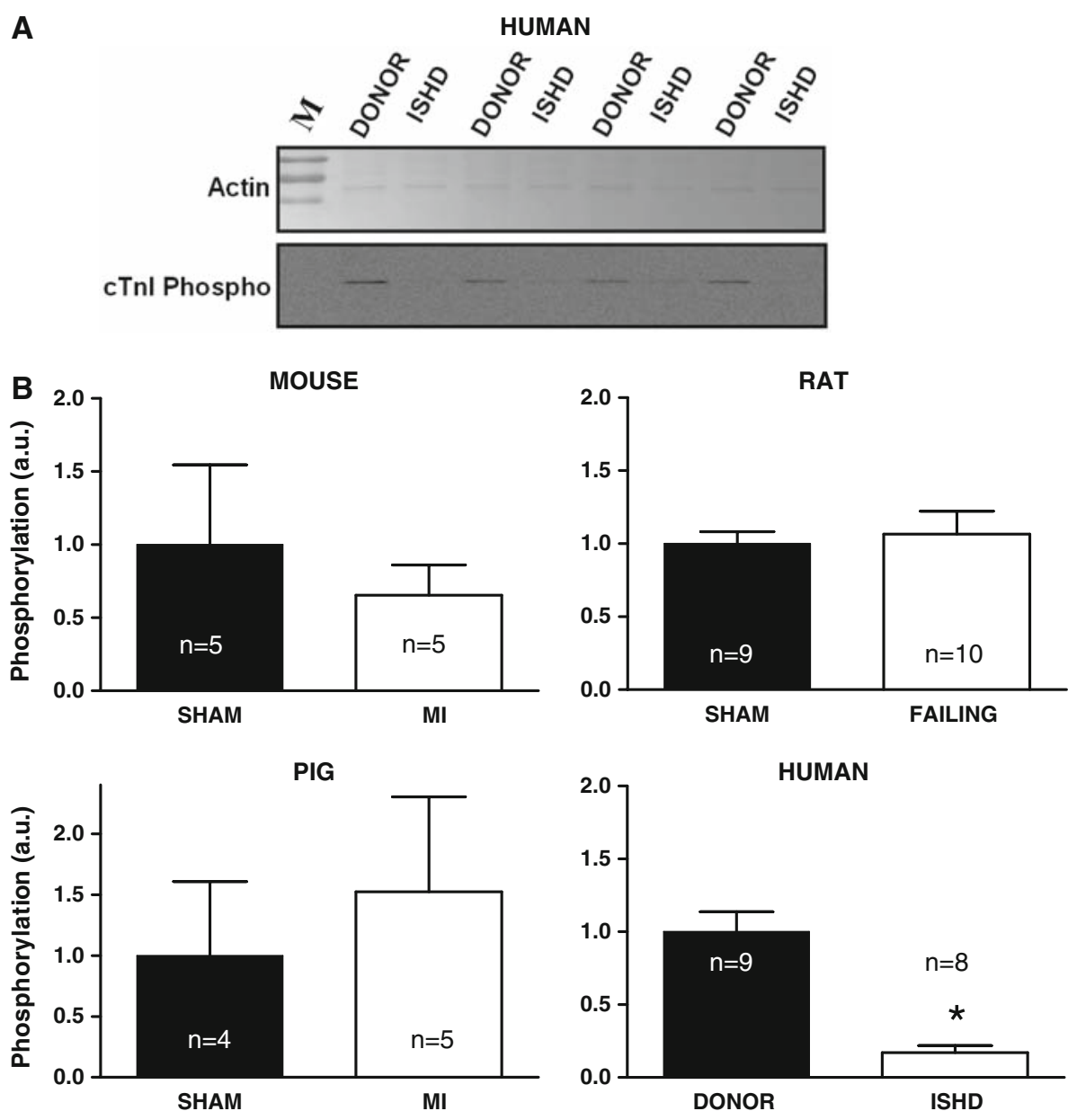
signalling in heart failure, reduced PKA-mediated phosphorylation would result in an enhanced myofilament $\mathrm{Ca}^{2+}$-sensitivity. Our functional measurements in single cardiomyocytes revealed an increase in myofilament $\mathrm{Ca}^{2+}$-sensitivity in all failing heart models (Fig. 2a), which could be corrected by exogenous PKA (Fig. 3). In vitro motility analysis of regulation of thin filament movement by troponin from failing and non-failing human myocardium revealed a similar increase in $\mathrm{Ca}^{2+}$-sensitivity in failing myocardium (Fig. 4a). Moreover, troponin regulation of thin filaments was similar when failing and non-failing human troponin was reconstituted with PKA-phosphorylated recombinant human cTnI (Fig. 4b). This indicates that reduced phosphorylation of the PKA-sites in cTnI (Ser 23/24) accounts for the enhanced myofilament $\mathrm{Ca}^{2+}$ sensivity observed in end-stage failing human myocardium.

The difference in $\mathrm{Ca}^{2+}$-sensitivity between failing and non-failing hearts was smallest in mice and largest in human. The relatively small difference between MI and sham mice (Fig. 2a) and small effects of PKA (Fig. 3a) may be explained by the relatively high baseline cTnI phosphorylation status (Fig. 6) in these animals. Defects in $\beta$-AR signalling may be difficult to uncover in mice due to the small dynamic range in response to $\beta$-AR stimulation. Although, the direction of the change in $\mathrm{pCa}_{50}$ was similar in all heart failure models, Bonferroni post-test analysis revealed that the difference in $\mathrm{pCa}_{50}\left(\Delta \mathrm{pCa}_{50}\right)$ between failing and non-failing cardiomyocytes was significantly larger in human compared to the mouse and rat model, while $\Delta \mathrm{pCa}_{50}$ (failing versus non-failing) did not differ from the pig model (Fig. 2c). Hence, large animal models may be more representative to investigate consequences of $\beta$-AR stimulation on myofilament protein phosphorylation and function.

Alternatively, the smaller differences in $\mathrm{pCa}_{50}$ in rodents and absence of reduced phosphorylation of $\beta$-AR target proteins in animal models in contrast to human, may be a reflection of disease progression, as the human studies involve end-stage heart failure. During earlier stages of heart failure, such as present in the animal studies, defects in $\beta$-AR signalling may be less severe, and the functional and proteomic phenotype observed in less severe stages of cardiac disease may be the resultant of changes in other signalling pathways as well. Within our human studies we observed a striking difference in cardiomyocyte stiffness, which may relate to severity of cardiac disease. In agreement with previous studies (Makarenko et al. 2004; Nagueh et al. 2004; Neagoe et al. 2002) cardiomyocyte stiffness in end-stage human heart failure was lower in comparison to non-failing donors (Fig. 5a). It has been proposed that the reduction in Fpas is due to a shift in titin isoform composition, from the stiff N2B to the more compliant N2BA isoform (Makarenko et al. 2004; Nagueh et al. 2004). In contrast, recent measurements in cardiomyocytes isolated from catheter biopsies from patients with less severe forms of heart failure (NYHA II-III) revealed enhanced cardiomyocyte stiffness in all heart failure patients, which was largely corrected with PKA (Borbély et al. 2005; van Heerebeek et al. 2006, 2008). In these samples, a similar shift in titin isoform composition was found as observed in end-stage heart failure. However, analysis of titin phosphorylation indicated differential titin isoform phosphorylation as possible cause for enhanced cardiomyocyte stiffness and may involve reduced protein kinase G-mediated phosphorylation (Krüger et al. 2007; Hamdani et al. 2007).

The functional consequences of impaired PKA-mediated phosphorylation may be balanced by changes in other kinases. In congestive heart failure models in rat (pressure overload and MI), reductions in Fmax and in $\mathrm{Ca}^{2+}$-sensitivity were associated with increased protein kinase $\mathrm{C}$ (PKC) expression (Belin et al. 2007). Increased phosphorylation of cTnI was observed in failing rat myocardium and deficits in myofilament function were corrected by phosphatase treatment. It has been proposed that the relative balance of phosphorylation of the $3 \mathrm{PKC}$ sites on cTnI (Ser 43/45 and Thr 144) is important for the regulation of its function (Solaro et al. 2008). Pseudophosphorylation of Ser 43/45 significantly reduced myofilament response to $\mathrm{Ca}^{2+}$ (Burkart et al. 2003) and phosphorylation of Thr 144 induced an increase in sensitivity to $\mathrm{Ca}^{2+}$ (Sumandea et al. 2003). Increased PKC activity and isoform expression has been reported in human heart failure (Bowling et al. 1999; Takeishi et al. 2000; Braz et al. 2004). However, it remains to be investigated if PKC targets the myofilament proteins in vivo (Huang and Walker 2004). Recent mass spectrometry analysis revealed a novel in vivo phosphorylation site in human cTnI (Ser 76/ Thr 77), which may be target of PKC (Zabrouskov et al. 2008). To establish if PKC-mediated protein phosphorylation impacts myofilament function in human cardiac disease quantitative mass spectrometry should be performed in cardiac samples, which are obtained under welldefined conditions, preferably before and after in vivo receptor stimulation.

\section{Reduced maximal force generating capacity}

The maximal force generating capacity of cardiomyocytes from remodelled myocardium early after a myocardial infarction was reduced and, unlike $\mathrm{pCa}_{50}$ and $\mathrm{Fpas}$, was not corrected by PKA. An important role has been assigned to PKC-mediated phosphorylation of cTnT at Thr 206 (Sumandea et al. 2003), and cTnI at Ser 43/45 (Burkart et al. 2003) in reducing myofilament Fmax. However, up to date, no changes in cTnT phosphorylation have been reported in 
any heart failure model (Fig. 6). Moreover, using an elegant troponin exchange method in failing and non-failing rat cardiomyocytes, Belin et al. (2006) showed that replacement of failing troponin by non-failing troponin restored myofilament $\mathrm{Ca}^{2+}$-sensitivity to values observed in non-failing cardiomyocytes, but not Fmax. Hence, alterations in troponin phosphorylation underlie changes in myofilament $\mathrm{Ca}^{2+}$-sensitivity, but are not the cause of reduced maximal force generating capacity.

In our previous study in MI mice (de Waard et al. 2007), exercise prevented the reduction in Fmax, which was associated with an increase in MLC2 phosphorylation, although MLC2 phosphorylation was not significantly reduced in MI (Fig. 6b, c). A transmural gradient in MLC2 phosphorylation has been described in rodent studies (Davis et al. 2001; Rajashree et al. 2005; Cazorla et al. 2005). A reduction in MLC2 phosphorylation in myocardium from MI mice may have been obscured by regional differences, as a significant reduction in MLC2 phosphorylation was observed in subendocardial tissue from MI pigs (Fig. 6b, c). Accordingly, a recent study in rats with ischaemic heart failure (Ait Mou et al. 2008) revealed impaired contractile function and reduced MLC2 phosphorylation only in subendocardial cardiomyocytes, which were both restored by exercise training. It has been proposed that the overall pattern of cardiac contraction depends on a spatial gradient of MLC2 phosphorylation (Davis et al. 2001), and loss of the MLC2 phosphorylation gradient may impair cardiac performance (Davis et al. 2001; Cazorla et al. 2005). Hence, myofilament function may be hampered by alterations in MLC2 phosphorylation. Investigation of transmural biopsies taken under wellcontrolled hemodynamic conditions allow careful analysis of MLC2 phosphorylation at rest and during enhanced cardiac load (i.e., upon receptor stimulation) and would allow investigation of the importance of a transmural MLC2 phosphorylation gradient for cardiac contraction in a large animal model. As the opposite changes in MLC2 phosphorylation in animal models with ischemic heart disease (reduction) and end-stage failing human ischemic cardiomyopathy (increase) may relate to severity of cardiac disease, changes during the progression of cardiac disease should be addressed as well.

In conclusion, cardiomyocyte force measurements revealed enhanced myofilament $\mathrm{Ca}^{2+}$-sensitivity and passive stiffness, which were both largely corrected with exogenous PKA indicative for hypo-phosphorylation of $\beta$ AR target proteins. When translated to in vivo cardiac performance, an enhancement in myofilament $\mathrm{Ca}^{2+}$-sensitivity and cardiomyocyte stiffness would both limit cardiac relaxation. This is illustrated by the positive correlation between high left ventricular end-diastolic pressures (LVEDP) in heart failure patients and high cardiomyocyte stiffness (Borbély et al. 2005). As high LVEDP could not be solely attributed to increased cardiac collagen, high LV filling pressures may be in part explained by high intrinsic stiffness of the cardiomyocytes. Though limiting relaxation, the enhanced myofilament $\mathrm{Ca}^{2+}$-sensitivity might increase cardiomyocyte force development and increase cardiac contractility.

Although the enhanced myofilament $\mathrm{Ca}^{2+}$-sensitivity in failing myocardium would enhance cardiomyocyte contractility, a reduction in Fmax would counteract this. This is illustrated by the relationship between absolute force development and $\mathrm{pCa}$ in Fig. $2 \mathrm{~b}$. In vivo, the impact of altered myofilament function depends on cytosolic $\left[\mathrm{Ca}^{2+}\right]$ during the different phases of the cardiac cycle. As systolic calcium levels are reduced in failing cardiomyocytes, it may be speculated that myofilament dysfunction in remodelled myocardium after MI further deteriorates cardiac performance.

\section{Future perspective}

Our data illustrate the need for careful tissue handling under well-defined conditions, preferably at the time of hemodynamic measurements, and careful analysis of the myofilament phosphoproteome. Research on the cellular mechanisms underlying human heart failure is hampered by the limited availability of biopsy material and, since biopsies are routinely obtained at one time point, by the lack of information on the dynamic aspects of the processes involved. The dynamic changes in cellular signalling critically depend on the phosphorylation status of a number of target proteins. Analysis of dynamic changes in intracellular signalling pathways and the interaction between kinases and phosphatases and their specific myofilament target proteins within intact cells is essential to establish a direct relation between changes in myofilament protein phosphorylation and myofilament dysfunction in heart failure.

Open Access This article is distributed under the terms of the Creative Commons Attribution Noncommercial License which permits any noncommercial use, distribution, and reproduction in any medium, provided the original author(s) and source are credited.

\section{References}

Aitt Mou Y, Reboul C, Andre L, Lacampagne A, Cazorla O (2008). Late exercise training improves non-uniformity of transmural myocardial function in rats with ischaemic heart failure. Cardiovasc Res [Epub ahead of print]

Belin RJ, Sumandea MP, Kobayashi T et al (2006) Left ventricular myofilament dysfunction in rat experimental hypertrophy and congestive heart failure. Am J Physiol Heart Circ Physiol 291:H2344-H2353. doi:10.1152/ajpheart.00541.2006 
Belin RJ, Sumandea MP, Allen EJ et al (2007) Augmented protein kinase $\mathrm{C}-\alpha$-induced myofilament protein phosphorylation contributes to myofilament dysfunction in experimental congestive heart failure. Circ Res 101:195-204. doi:10.1161/CIRCRESAHA.107.148288

Bers DM (2002) Cardiac excitation-contraction coupling. Nature 415:198-205. doi:10.1038/415198a

Bohm M, Maack C (2000) Treatment of heart failure with betablockers. Basic Res Cardiol 95:I15-I24. doi:10.1007/s0039500 70004

Borbély A, van der Velden J, Bronzwaer JGF et al (2005) Cardiomyocyte stiffness in diastolic heart failure. Circulation 111: 774-781. doi:10.1161/01.CIR.0000155257.33485.6D

Bowling N, Walsh RA, Song G et al (1999) Increased protein kinase $\mathrm{C}$ activity and expression of $\mathrm{Ca} 2+$-sensitive isoforms in the failing human heart. Circulation 99:384-391

Braz JC, Gregory K, Pathak A et al (2004) PKC-alpha regulates cardiac contractility and propensity toward heart failure. Nature $10: 248-254$

Bristow MR (2000) Beta-adrenergic receptor blockade in chronic heart failure. Circulation 101:558-569

Burkart EM, Sumandea MP, Kobayashi T et al (2003) Phosphorylation or glutamic acid substitution at protein kinase $\mathrm{C}$ sites on cardiac troponin I differentially depress myofilament tension and shortening velocity. J Biol Chem 278:11265-11272. doi:10.1074/jbc.M210712200

Cazorla O, Szilagyi S, Le Guennec JY et al (2005) Transmural stretch-dependent regulation of contractile properties in rat hearts and its alteration after myocardial infarction. FASEB J 19:88-90

Cazorla O, Szilagyi S, Vignier N et al (2006) Length and protein kinase A modulations of myocytes in cardiac myosin binding protein C-deficient mice. Cardiovasc Res 69:370-380. doi:10.1016/j.cardiores.2005.11.009

Cohn JN, Levine TB, Olivari MT et al (1984) Plasma norepinephrine as a guide to prognosis in patients with chronic congestive heart failure. N Engl J Med 311:819-823

Davis JS, Hassanzadeh S, Winitsky S et al (2001) The overall pattern of cardiac contraction depends on a spatial gradient of myosin regulatory light chain phosphorylation. Cell 107:631-641. doi:10.1016/S0092-8674(01)00586-4

De Waard MC, van der Velden J, Bito V et al (2007) Early exercise training normalizes myofilament function and attenuates left ventricular pump dysfunction in mice with a large myocardial infarction. Circ Res 100:1079-1088. doi:10.1161/ 01.RES.0000262655.16373.37

Édes IF, Tóth A, Csányi G et al (2008) Late-stage alterations in myofibrillar contractile function in a transgenic mouse model of dilated cardiomyopathy (Tg $\alpha$ q*44). J Mol Cell Cardiol 45:363372. doi:10.1016/j.yjmcc.2008.07.001

Fraser IDC, Marston SB (1995) In vitro motility analysis of actintropomyosin regulation by troponin and $\mathrm{Ca}^{2+}$ : the thin filament is switched as a single cooperative unit. J Biol Chem 270:78367841. doi:10.1074/jbc.270.34.20156

Fukuda N, Wu Y, Nair P et al (2005) Phosphorylation of titin modulates passive stiffness of cardiac muscle in a titin isoformdependent manner. J Gen Physiol 125:257-271. doi:10.1085/ jgp.200409177

Hamdani N, Borbely A, Boontje NM et al (2007) Protein kinase G corrects high cardiomyocyte resting tension in diastolic heart failure. Circulation 116:II-708 (abstract)

Hamdani N, Kooij V, Merkus D et al (2008) Sarcomeric dysfunction in heart failure. Cardiovasc Res 77:649-658. doi:10.1093/cvr/ cvm079

Harding SE, Brown LA, Wynne DG et al (1994) Mechanisms of beta adrenoceptor desensitisation in the failing human heart. Cardiovasc $\operatorname{Res} 28: 1451-1460$. doi:10.1093/cvr/28.10.1451
Huang X, Walker JW (2004) Myofilament anchoring of protein kinase C-epsilon in cardiac myocytes. J Cell Sci 117:1971-1978. doi: $10.1242 /$ jcs. 01044

Kentish JC, McCloskey DT, Layland J et al (2001) Phosphorylation of troponin I by protein kinase A accelerates relaxation and crossbridge cycle kinetics in mouse ventricular muscle. Circ Res 88:1059-1065. doi:10.1161/hh1001.091640

Knott A, Purcell IF, Marston SB (2002) In vitro motility analysis of thin filaments from failing and non-failing human hearts induces slower filament sliding and higher $\mathrm{Ca}^{2+}$-sensitivity. J Mol Cell Cardiol 34:469-482. doi:10.1006/jmcc.2002.1528

Kobayashi T, Solaro RJ (2005) Calcium, thin filaments, and the integrative biology of cardiac contractility. Annu Rev Physiol 67:39-67. doi:10.1146/annurev.physiol.67.040403.114025

Korstjens IJ, Rouws CH, van der Laarse WJ et al (2002) Myocardial force development and structural changes associated with monocrotaline induced cardiac hypertrophy and heart failure. J Muscle Res Cell Motil 23:93-102. doi:10.1023/A:1019988815436

Krüger M, Linke WA (2006) Protein kinase-A phosphorylates titin in human heart muscle and reduces myofibrillar passive tension. J Muscle Res Cell Motil 27:435-444. doi:10.1007/s10974-0069090-5

Krüger M, dos Remedios C, Linke WA (2007) Titin phosphorylation by protein kinases $\mathrm{A}$ and $\mathrm{G}$ in normal and failing human hearts decreases myocardial passive stiffness. Circulation 116:II-301 (abstract)

Lamberts RR, Soekhoe TW, Hamdani N et al (2007) Frequencydependent $\mathrm{Ca}^{2+}$-desensitization in failing rat hearts. J Physiol 582:695-709. doi:10.1113/jphysiol.2007.134486

Leineweber K, Seyfarth T, Brodde OE (2000) Chamber-specific alterations of noradrenaline uptake (uptake 1 ) in right ventricles of monocrotaline-treated rats. Br J Pharmacol 131:1438-1444. doi:10.1038/sj.bjp.0703698

Makarenko I, Opitz CA, Leake MC et al (2004) Passive stiffness changes caused by upregulation of compliant titin isoforms in human dilated cardiomyopathy hearts. Circ Res 95:708-716. doi:10.1161/01.RES.0000143901.37063.2f

Messer AE, Jacques AM, Marston SB (2007) Troponin phosphorylation and regulatory function in human heart muscle: dephosphorylation of ser23/24 on troponin I could account for the contractile defect in end-stage heart failure. J Mol Cell Cardiol 42:247-259. doi:10.1016/j.yjmcc.2006.08.017

Nagueh SF, Shah G, Wu Y et al (2004) Altered titin expression, myocardial stiffness, and left ventricular function in patients with dilated cardiomyopathy. Circulation 110:155-162. doi:10.1161/01.CIR.0000135591.37759.AF

Neagoe C, Kulke M, del Monte F et al (2002) Titin isoform switch in ischemic human heart disease. Circulation 106:1333-1341. doi:10.1161/01.CIR.0000029803.93022.93

Packer M (1995) Evolution of the neurohormonal hypothesis to explain the progression of chronic heart failure. Eur Heart $\mathrm{J}$ 16:F4-F6

Rajashree R, Blunt BC, Hofmann PA (2005) Modulation of myosin phosphatase targeting subunit and protein phosphatase 1 in the heart. Am J Physiol 289:H1736-H1743

Solaro RJ, Moir AJ, Perry SV (1976) Phosphorylation of troponin I and the inotropic effect of adrenaline in the perfused rabbit heart. Nature 262:615-617. doi:10.1038/262615a0

Solaro RJ, Rosevear P, Kobayashi T (2008) The unique functions of cardiac troponin I in the control of cardiac muscle contraction and relaxation. Biochem Biophys Res Commun 369:82-87. doi:10.1016/j.bbrc.2007.12.114

Stelzer JE, Patel JR, Walker JW, Moss RL (2007) Differential roles of cardiac myosin-binding protein $\mathrm{C}$ and cardiac troponin $\mathrm{I}$ in the myofibrillar force responses to protein kinase A phosphorylation. Circ Res 101:503-511. doi:10.1161/CIRCRESAHA.107.153650 
Sumandea MP, Pyle WG, Kobayashi T et al (2003) Identification of a functionally critical protein kinase $\mathrm{C}$ phosphorylation residue of cardiac troponin T. J Biol Chem 278:35135-35144. doi:10.1074/ jbc.M306325200

Takeishi Y, Jalili T, Hoit BD et al (2000) Alterations in $\mathrm{Ca}^{2+}$ cycling proteins and $G$ alpha q signaling after left ventricular assist device support in failing human hearts. Cardiovasc Res 45:883888

Van der Velden J, Papp Z, Zaremba R et al (2003) Increased $\mathrm{Ca}^{2+}$. sensitivity of the contractile apparatus in end-stage human heart failure results from altered phosphorylation of contractile proteins. Cardiovasc Res 57:37-47. doi:10.1016/S0008-6363(02)00606-5

Van der Velden J, Merkus D, Klarenbeek BR et al (2004) Alterations in myofilament function contribute to left ventricular dysfunction in pigs early after myocardial infarction. Circ Res 95:e85e95. doi:10.1161/01.RES.0000149531.02904.09

Van Heerebeek L, Borbely A, Niessen HW et al (2006) Myocardial structure and function differ in systolic and diastolic heart failure. Circulation 113:1966-1973. doi:10.1161/CIRCULATION AHA.105.587519

Van Heerebeek L, Hamdani N, Handoko L et al (2008) Diastolic stiffness of the failing diabetic heart: importance of fibrosis, advanced glycation endproducts and myocyte resting tension. Circulation 117:52-60. doi:10.1161/CIRCULATIONAHA.107.728550

Van Kats JP, Duncker DJ, Haitsma DB et al (2000) Angiotensinconverting enzyme inhibition and angiotensin II type 1 receptor blockade prevent cardiac remodeling in pigs after myocardial infarction: role of tissue angiotensin II. Circulation 102:15561563

Wolff MR, Buck SH, Stoker SW et al (1996) Myofibrillar calcium sensitivity of isometric tension is increased in human dilated cardiomyopathies. J Clin Invest 98:167-176. doi:10.1172/ JCI118762

Zabrouskov V, Ge Y, Schwartz J, et al. (2008). Unraveling molecular complexity of phosphorylated human cardiac troponin I by top down electron capture dissociation/electron transfer dissociation mass spectrometry. Mol Cell Proteomics 7:1838-1849

Zaremba R, Merkus D, Hamdani N et al (2007) Quantitative analysis of myofilament protein phosphorylation in small cardiac biopsies. Proteom Clin Applic 1:1285-1290. doi:10.1002/prca.200600891

Zhang R, Zhao J, Mandveno A et al (1995) Cardiac troponin I phosphorylation increases the rate of cardiac muscle relaxation. Circ Res 76:1028-1035 